\title{
Our expert highlights the most important research articles across the spectrum of topics relevant to the field of genetics and pathology of breast cancer
}

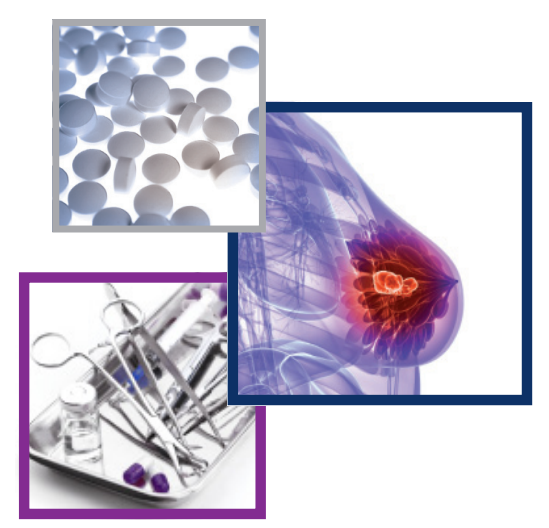

Expert: Hitoshi Tsuda, National Cancer Center Hospital, Tokyo, Japan

\section{Asmann YW, Necela BM, Kalari KR et al. Detection of redundant fusion transcript as biomarkers or disease- specific therapeutic targets in breast cancer. Cancer Res. 72(8), 1921-1928 (2012).}

Genomic rearrangements (e.g., chromosome translocations, inversions and/or interstitial deletions) can give rise to fusion gene products. Recently, these gene products have begun to be appreciated not only in hematopoietic malignancies and soft tissue tumors, but also in common solid tumors (e.g., lung and prostate cancers). In this study, a number of novel fusion transcripts were detected from 24 breast cancer tissues by means of integrated genome/transcriptome analyses and bioinformatics. Among the 45 redundant (distributed among multiple tumors) tumor-subtype specific fusion transcripts identified, numbers 7 and 8 were unique to estrogen receptor-positive tumors and triplenegative (estrogen receptor-, progesterone receptor- and HER2-negative) tumors, respectively. The genomic rearrangements that caused these fusion transcripts occurred most frequently in chromosomes 1, 2, 17 and 19 , and two regions in chromosome 19 (19p12-p13 or 19q13) seemed to be hot-spots for triple-negative tumors. These fusion gene products are expected to provide therapeutic targets and/or clinically relevant tumor biomarkers.

Shah SP, Roth A, Goya R et al. The clonal and mutational evolution spectrum of primary triple-negative breast cancers. Nature 486(7403), 395-399 (2012).
Triple-negative (estrogen receptor-, progesterone receptor- and HER2-negative) breast cancers (TNBCs) have been shown to be heterogeneous in terms of histopathological, immunohistochemical and biological features. The present study performed integrated genome/transcriptome analyses in 104 surgically resected TNBCs. Mutations were found frequently in $p 53$ (43-62\%), PIK3CA (10\%), USH2A (9.2\%), MYO3A (9.2\%), PTEN (7.7\%) and RBI (7.7\%), but were also found sparsely in various genes. Pathways affected by mutations consisted not only of those associated with driver mutations (e.g., p53-related, chromatin remodeling, PI3K signaling and ERBB signaling), but also in those associated with cytoskeletal structure, cell shape and motility. Individual TNBCs were heterogeneous in terms of gene mutation composition and clonal frequency modes. Mutations of known driver genes ( $p 53$, PIK3CA and $P T E N)$ had among the highest clonal frequencies and were clearly early events, whereas mutations in cell shape/motility and extracellular matrix signaling that appeared in the lower clonal frequency groups were likely to occur due to additional events during tumor progression. These data will help clarify the molecular basis of phenotypic and biological heterogeneity in TNBCs.

Curtis C, Shah SP, Chin S-F et al. The genomic and transcriptomic architecture of 2,000 breast tumours reveals novel subgroups. Nature 486(7403), 346-352 (2012).

This study discovered that breast cancers were able to be classified into ten novel

\section{News \& Views}

News

Journal Watch Interview

Conference Scenes 
subgroups from the integrated 'landscape' based on data from integrated genome/transcriptome analyses. By combination with outlying mRNA expression profiles, somatic copy number aberrations (CNAs) were divided into those acting in cis, which have an impact on their own expression, and those acting in trans, which are associated with genes at other genomic sites. CNAs acting in cis included known drivers (ZHF703, PTEN, MYC, CCND1, MDM2, ERBB2 [HER2] and $C C N E 1)$ and dominated the expression landscape. A total of 1992 tumors were classified into ten groups (integrative clusters or IntClust 1-10) that were correlated with patients' clinical outcomes: the high-risk group comprised IntClust 2 (11q13/14 cis-acting CNA, luminal) and 5 (ERBB2-amplified); the intermediate-risk group comprised IntClust $1(17 \mathrm{q} 23 / 20 \mathrm{q}$ cis-acting CNA, luminal-B), 6 (8p12 cis-acting CNA, luminal), 9 (8q cis-acting CNA/20q-amplified mixed) and 10 (basal-like, cis-acting 5-loss/8q-gain/10pgain/12p-gain); and the favorable group comprised IntClust 3 (paucity of CNAs, invasive lobular and tubular carcinomas), 4 ('CNA-devoid', estrogen receptor-positive and -negative), 8 (classical 1q-gain/16qloss) and 7 (16p-gain/16q-loss with $8 \mathrm{q}$-amplification).

Rizzo M, Linebarger J, Lowe MC et al. Management of papillary breast lesions diagnosed on core-needle biopsy: clinical pathologic and radiologic analysis of 276 cases with surgical follow-up. J. Am. Coll. Surg. 214(3), 280-287 (2012).

The management of benign intraductal papilloma (IDP) without atypia, as diagnosed on core needle biopsy (CNB), remains controversial. In this study, in 276 patients with papillary breast lesions diagnosed as solitary IDP on CNB, the rates of upgraded diagnosis after excision were examined retrospectively. Of 234 IDPs without atypia on the CNB, $63(27 \%)$ upgraded to a lesion of greater clinical significance, 42 to atypical ductal hyperplasia and 21 to ductal carcinoma in situ or invasive ductal carcinoma. Of 43 IDPs with atypical ductal hyperplasia/atypical lobular hyperplasia on the CNB, 16 (38\%) upgraded to ductal carcinoma in situ or invasive ductal carcinoma. Older age was the only predictive factor, and no radiological parameters were predictive of upgrade. It was concluded that surgical excision should be performed for all IDPs to delineate subsequent clinical management. Lack of information of number of CNB cores, deviation in the patient population and so on were limitations with this study.

Rendi MH, Dintzis SM, Lehman CD et al. Lobular in-situ neoplasia on breast core needle biopsy: imaging indication and pathologic extent can identify which patients require excisional biopsy. Ann. Surg. Oncol. 19(3), 914-921 (2012).
The management of lobular neoplasia (LN), including lobular carcinoma in situ and atypical lobular hyperplasia, identified by core needle biopsy (CNB) is currently variable. Multiple studies from different institutions yielded upgrade rates ranging from 1 to $35 \%$. In this retrospective study, the rates of upgraded diagnosis after excision were examined retrospectively in $93 \mathrm{LNs}$ identified on CNB. Cases with carcinoma or pleomorphic lobular carcinoma in situ in the same biopsy site were excluded. Of 68 cases of $\mathrm{LN}$ alone on $\mathrm{CNB}$, only $4.4 \%$ upgraded to carcinoma, whereas of 25 cases of LN-positive atypical ductal hyperplasia, $16 \%$ upgraded. The decision to excise isolated LNs when found on CNB should depend on multiple factors. The authors' algorithm allowed for close follow-up of patients with calcifications on routine screening mammography and isolated LNs on CNB for normal-risk patients and when fewer than four foci of LNs and no other high-risk lesions were present. All other cases should be recommended for excisional biopsy.

\section{Financial \& competing interests}

\section{disclosure}

The author has no relevant affiliations or financial involvement with any organization or entity with a financial interest in or financial conflict with the subject matter or materials discussed in the manuscript. This includes employment, consultancies, honoraria, stock ownership or options, expert testimony, grants or patents received or pending, or royalties.

No writing assistance was utilized in the production of this manuscript.

Journal Watch highlights some of the most important papers recently published in the field of breast cancer management and research. The editorial team welcomes recommendations for relevant papers for inclusion in future issues.

Please direct your suggestions to:

Joanna Milburn, Commisioning Editor, Breast Cancer Management

j.milburn@futuremedicine.com 